\title{
Resolving debris discs in the far-infrared: Early highlights from the DEBRIS survey ${ }^{\star}$
}

\author{
B. C. Matthews ${ }^{1,2}$, B. Sibthorpe ${ }^{3}$, G. Kennedy ${ }^{4}$, N. Phillips ${ }^{5}$, L. Churcher ${ }^{4}$, G. Duchêne ${ }^{6,7}$, J. S. Greaves ${ }^{8}$, \\ J.-F. Lestrade ${ }^{9}$, A. Moro-Martin ${ }^{10,11}$, M. C. Wyatt ${ }^{4}$, P. Bastien ${ }^{12}$, A. Biggs ${ }^{3}$, J. Bouvier ${ }^{6}$, H. M. Butner ${ }^{13}$, \\ W. R. F. Dent ${ }^{14}$, J. Di Francesco ${ }^{1,2}$, J. Eislöffel ${ }^{15}$, J. Graham ${ }^{7}$, P. Harvey ${ }^{16}$, P. Hauschildt ${ }^{17}$, W. S. Holland ${ }^{3}$, J. Horner ${ }^{18}$, \\ E. Ibar ${ }^{3}$, R. J. Ivison ${ }^{3,5}$, D. Johnstone ${ }^{1,2}$, P. Kalas ${ }^{7}$, J. Kavelaars ${ }^{1,2}$, D. Rodriguez ${ }^{19}$, S. Udry ${ }^{20}$, P. van der Werf ${ }^{21}$, \\ D. Wilner ${ }^{22}$, and B. Zuckerman ${ }^{19}$ \\ (Affiliations are available in the online edition)
}

Received 31 March 2010 / Accepted 9 May 2010

\begin{abstract}
We present results from the earliest observations of DEBRIS, a Herschel key programme to conduct a volume- and flux-limited survey for debris discs in A-type through M-type stars. PACS images (from chop/nod or scan-mode observations) at 100 and $160 \mu \mathrm{m}$ are presented toward two A-type stars and one F-type star: $\beta$ Leo, $\beta$ UMa and $\eta$ Corvi. All three stars are known disc hosts. Herschel spatially resolves the dust emission around all three stars (marginally, in the case of $\beta \mathrm{UMa}$ ), providing new information about discs as close as $11 \mathrm{pc}$ with sizes comparable to that of the Solar System. We have combined these data with existing flux density measurements of the discs to refine the SEDs and derive estimates of the fractional luminosities, temperatures and radii of the discs.
\end{abstract}

Key words. circumstellar matter - stars: individual: $\beta$ Leo - infrared: stars - stars: individual: $\beta$ UMa - stars: individual: $\eta$ Corvi

\section{Introduction}

Debris discs are flattened distributions of planetesimals and dust located at radii of 1-1000 AU around main-sequence stars (see (see Wyatt 2008, for a recent review). The dust cannot be primordial since its lifetime in orbit is significantly less than the age of the host stars. Instead, dust is replenished from a population of colliding km-sized planetesimals (Wyatt \& Dent 2002; Thébault $\&$ Augereau 2007). Over time, the dust distribution is shaped by any planetary-sized bodies in the system (e.g., Dominik \& Decin 2003; Wyatt et al. 2007). Therefore, resolved images of discs constrain models of the structure and evolution of planetary systems.

Far-infrared and submillimetre observations are the best way to search for dust around nearby stars due to the favorable contrast of the disc relative to the star. At these wavelengths, the disc emission is optically thin and is sensitive to the large (up to $\sim 1 \mathrm{~mm}$ ), cold grains which dominate the disc's dust mass.

The Herschel Space Observatory offers three major advantages for the detection and characterization of debris discs: farinfrared sensitivity, angular resolution and wavelength coverage. With its $3.5 \mathrm{~m}$ mirror, its sensitivity at far-infrared wavelengths is superior to any previous instrument. With its resolution of 6.'7 at $100 \mu \mathrm{m}$, Herschel has the potential to resolve many debris discs, particularly toward nearby stars. Finally, with detectors at $100,160,250,350$ and $500 \mu \mathrm{m}$, Herschel has the means to

* Herschel is an ESA space observatory with science instruments provided by European-led Principal Investigator consortia and with important participation from NASA. sample the spectral energy distribution (SED) of disc emission across the peak, meaning models can be better constrained even for discs which are not resolved.

DEBRIS (Disc Emission via a Bias-free Reconnaissance in the Infrared/Submillimetre) is an open time key programme which uses PACS (Photodetector Array Camera and Spectrometer) and (for appropriate targets) SPIRE (Spectral and Photometric Imaging REceiver) to detect, resolve and characterize debris discs around a volume-limited sample of $446 \mathrm{~A}$ through $\mathrm{M}$ type stars. The goals of DEBRIS include establishing the incidence and evolution of debris discs as a function of stellar type, age, multiplicity, etc.; the characterization of discs in terms of size, temperature, dust mass and morphology (where the disc asymmetries could indicate the presence of planetary companions); and the understanding of our own Solar System in the context of the larger debris disc population. Full details of the DEBRIS survey and goals will be presented in a forthcoming paper (B. Matthews et al. 2010, in preparation). Here, we present PACS observations toward three of the first targets of the DEBRIS survey. We briefly summarize the observations and targets in Sect. 2, present the results in Sect. 3 and discuss three sources in detail in Sect. 4. We summarize the paper in Sect. 5.

\section{Observations and data reduction}

DEBRIS is a flux-limited survey and as such it observes each target to a uniform depth $\left(1.2 \mathrm{mJy}^{\text {beam }^{-1}}\right.$ at $\left.100 \mu \mathrm{m}\right)$, resulting in different mass limits for targets at different distances and of different stellar spectral types. Here, we present 100 and $160 \mu \mathrm{m}$ photometry observations toward three nearby stars (see Table 1) 
Table 1. Stellar and disc parameters.

\begin{tabular}{lccc}
\hline \hline Parameter & $\beta$ Leo & $\beta$ UMa & $\eta$ Corvi \\
\hline UNS ID & A005 & A024 & F063 \\
HD number & HD 102647 & HD 95418 & HD 109085 \\
Spec. Type & A3 Va & A1 V & F2 V \\
PACS OM & scan & point-source & scan \\
$D$ [pc] & 11.0 & 24.3 & 18.2 \\
$\mathrm{rms}_{100}[\mathrm{mJy} / \mathrm{beam}]$ & 1.4 & 3.1 & 1.2 \\
$\mathrm{rms}_{160}[\mathrm{mJy} / \mathrm{beam}]$ & 3.9 & 7.6 & 5.0 \\
Aperture [arcsec] & 20 & 15 & 20 \\
$F_{100}[\mathrm{mJy}]$ & $500 \pm 50$ & $390 \pm 39$ & $300 \pm 30$ \\
$F_{160}[\mathrm{mJy}]$ & $230 \pm 46$ & $120 \pm 25$ & $290 \pm 58$ \\
\hline$F W H M_{\text {min }}^{100}\left[{ }^{\prime \prime}\right]$ & $9.2 \pm 0.1$ & $7.2 \pm 0.1$ & $16.4 \pm 0.4$ \\
$F W H M_{\text {maj }}^{100}\left[{ }^{\prime \prime}\right]$ & $10.4 \pm 0.1$ & $8.4 \pm 0.2$ & $18.3 \pm 0.4$ \\
$P A^{100}\left[{ }^{\circ}\right]$ & $125 \pm 3$ & $114 \pm 5$ & $102 \pm 7$ \\
$L^{*}\left[L_{\odot}\right]$ & 13.5 & 60 & 5.0 \\
$T_{\text {eff }}^{*}[\mathrm{~K}]$ & 8380 & 9340 & 6950 \\
$f_{D}=L_{I R} / L^{*}$ & $2.2 \times 10^{-5}$ & $1.4 \times 10^{-5}$ & $3.6 \times 10^{-4}$ \\
$T_{\text {disc }}[\mathrm{K}]$ & 112 & 109 & 31,354 \\
$R_{\text {dust }}[\mathrm{AU}]$ & 23 & 51 & $174,1.4$ \\
$R_{\text {obs }}{ }^{a}[\mathrm{AU}]$ & $\sim 39$ & $\sim 47$ & $\sim 145$ \\
\hline
\end{tabular}

Notes. ${ }^{(a)}$ Estimates of radius are deconvolved from the beam, assuming $R_{\text {obs }} / D=\operatorname{sqrt}\left(F W H M_{\text {min }}^{100} \times F W H M_{\text {maj }}^{100}-\mathrm{BEAM}^{2}\right)$.

performed with the ESA Herschel Space Observatory (Pilbratt et al. 2010) utilizing the PACS (Poglitsch, Waelkens \& Geis 2010) instrument. The results presented here were taken during early testing phases or during the science demonstration phase on Herschel (2009 Sept. - Dec.)

The images were obtained using two different observing strategies: point source chop/nod, and small scan-map modes (see the PACS observers' manual ${ }^{1}$ ). For point-source mode observations, seven contiguous repeat chop/nod observations were performed. Scan map observations had eight repeats in a single scan direction at a rate of $20^{\prime \prime} / \mathrm{s}$. Four $3^{\prime}$ scan legs were performed per map with a $2^{\prime \prime}$ separation between legs. The total observing times were 1072 and 1220 s, respectively, for each chop/nod and scanning observation.

Table 1 shows the survey ("UNS") identifier for each target as well as the source and observing details. Phillips et al. (2010) contains details of the development of the Unbiased Nearby Stars sample from which the DEBRIS targets are drawn.

These data were reduced using the Herschel interactive processing environment (HIPE Ott 2010). Maps were obtained via the default PACS naïve map-making methods photProject and photProjectPointSource in HIPE for the scanning and point source observing modes respectively. Scanned data were prefiltered to remove low frequency $(1 / f)$ noise using a boxcar filter with width equal to 1,5 . All bright sources in the map were masked prior to filtering to avoid filter ringing type artefacts. The chop/nod configuration meant that no equivalent filtering was required for the data obtained in point source mode.

All three targets presented in this paper are shared targets with the DUNES Herschel key programme (PI: C. Eiroa) which has science goals complementary to those of DEBRIS. Details on the distribution of targets will be discussed in a survey description paper (B. Matthews 2010, in preparation).

\footnotetext{
${ }_{1}$ http://herschel.esac . esa. int/Docs/PACS/html/pacs_om. html
}

\section{Results}

Figure 1 shows the 100 and $160 \mu \mathrm{m}$ images for the three targets. The rms levels achieved in each observation are summarized in Table 1. The higher noise levels associated with the point-source mode are evident. The scan map noise levels were significantly lower for comparable observing times and, for $\eta$ Corvi, meet the DEBRIS rms specifications. For $\beta$ Leo, the rms is higher by $\sim 15 \%$.

The integrated flux densities are estimated for each image (star + disc). This is done with simple aperture photometry using apertures (see Table 1) centred on the peak emission. Poglitsch, Waelkens \& Geis (2010) detail the flux calibration of PACS data and estimate the calibration uncertainties in the measured flux densities to be $10 \%$ and $20 \%$ for 100 and $160 \mu \mathrm{m}$, respectively. The dominant flux calibration uncertainties have been combined in quadrature with statistical uncertainties from the rms levels in the maps. These combined uncertainties are applied to the fluxes in Table 1.

The flux densities reported in Table 1 are plotted on spectral energy distributions in Fig. 2. The disc components of $\beta$ Leo and $\beta$ UMa are well fit by a simple blackbody in the absence of submillimetre detections, but $\eta$ Corvi requires a two component fit to its disc emission: a warmer blackbody and a modified blackbody for the cold component to fit the submillimetre flux densities. The temperature, radius $\left(R_{\text {dust }}\right)$ and fractional luminosity of these fits are reported in Table 1.

Fitting of 2D Gaussians to each source at $100 \mu \mathrm{m}$ yields $F W H M$ values (see Table 1) larger than the nominal PACS PSF of 6.'7. Analysis of an observation of Vesta yields a PSF of 6.'6 $\times 6$ '. 9 . Vesta is a cool blackbody for which the response within the $100 \mu \mathrm{m}$ filter should be very similar to our dust discs. It has a temperature measured in the submillimetre at 130-160 K (Chamberlain et al. 2007), slightly warmer than our two A-star dist discs at $\sim 110 \mathrm{~K}$. Taking into account the spectral response theoretically, the range of $F W H M$ varies by less than $5 \%$, for slopes from -2 to +1 in $\lambda F_{\lambda}$. Larger PSFs have been measured yielding maxiumum dimensions as high as $77^{\prime \prime} 3$. Since only the long axis of the $\beta$ UMa disc exceeds this size, we claim this disc is marginally resolved. The $\beta$ Leo and $\eta$ Corvi discs are well resolved at $100 \mu \mathrm{m}$. Estimates of deconvolved disc radius have been made from the $F W H M$. We call this radius estimate $R_{\mathrm{obs}}$ (Table 1).

\section{Discussion}

\section{1. $\beta$ Leo}

Figure 1 shows the first resolved images of the disc around $\beta$ Leo. The blackbody temperature and dust luminosity results given in Table 1 are consistent with the values found in previous works (Su et al. 2006; Holmes et al. 2003). The fractional dust luminosity of $2.3 \times 10^{-5}$ is $15 \%$ higher than the estimate from $\mathrm{Su}$ et al. (2006). The increase is due to a slight increase in dust to match the PACS flux densities.

The radius estimates found for $\beta$ Leo are comparable to that of the Kuiper belt ( $\sim 50 \mathrm{AU})$. This makes the $\beta$ Leo disc one of the smallest disc radii yet resolved at any wavelength (see, for instance, the "Circumstellar Disks Database"2) although smaller characteristic orbital radii have been derived based on single temperature blackbody fits to the dust components (e.g., Rhee et al. 2007).

\footnotetext{
${ }^{2}$ circumstellardisks.org
} 

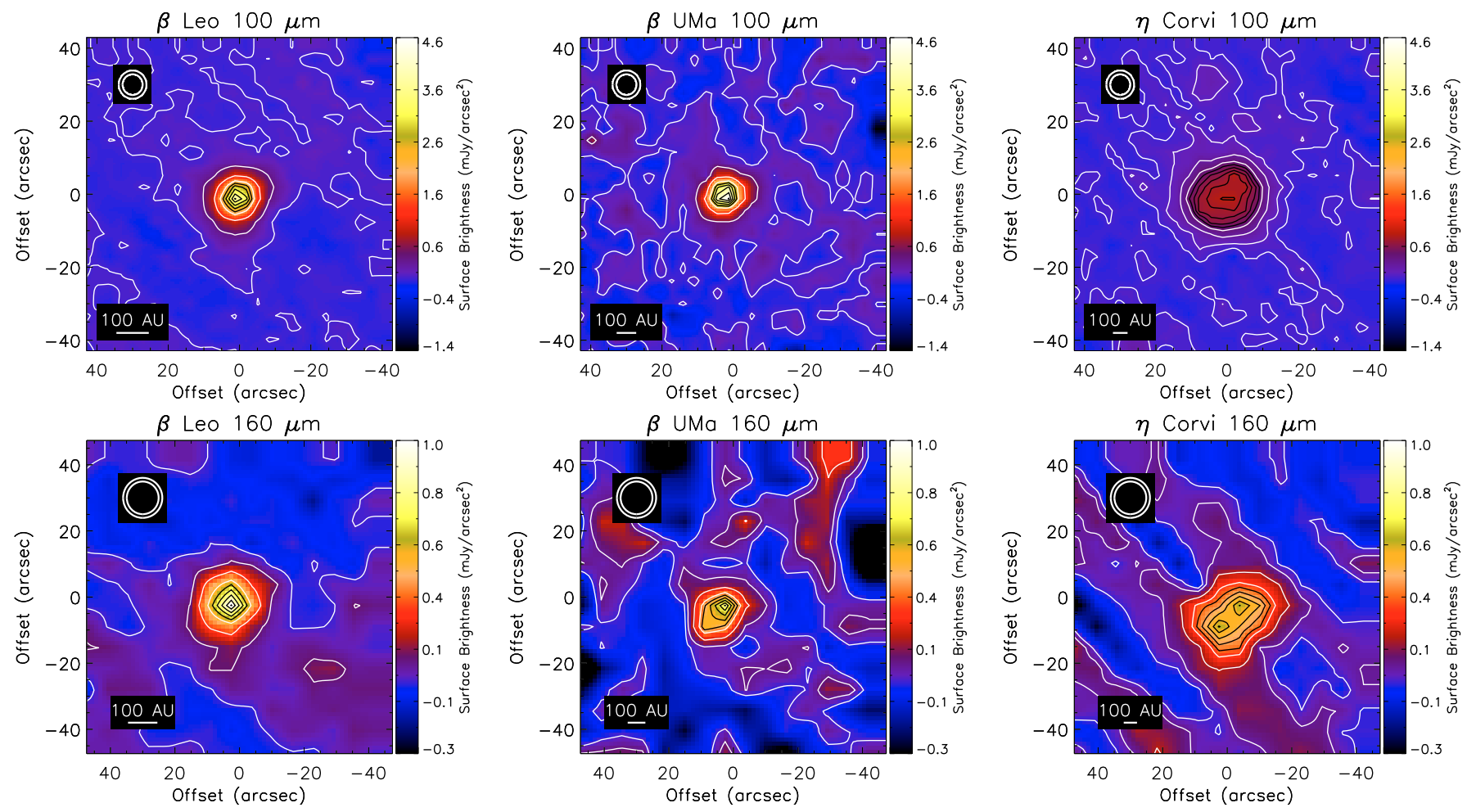

Fig. 1. Images of the 100 and $160 \mu \mathrm{m}$ emission from three DEBRIS targets: $\beta$ Leo, $\beta$ UMa and $\eta$ Corvi. Contours are shown at $0,10,30,50,60$, 70, 80, 90 and $99 \%$ of the peak in each map. The 1- $\sigma$ rms noise levels are given in Table 1. Circles in the upper left corner of each panel mark the nominal beam sizes for a scan speed of $20^{\prime \prime} / \mathrm{s}$, i.e., 6."7 and $11^{\prime \prime}$ at 100 and $160 \mu \mathrm{m}$, respectively. The negative images created by the chop/nod observing mode are visible in the $\beta$ UMa images. Striping in the $\eta$ Corvi image at $160 \mu \mathrm{m}$ is due to high $1 / \mathrm{f}$ noise and filtering artefacts.

The difference between $R_{\mathrm{obs}}$ and $R_{\text {dust }}$ provides an opportunity to learn about the grains within this disc. Because differently sized grains can have the same temperature at different distances from a star, the SED models in Fig. 2 are degenerate. This degeneracy is broken by the resolved imaging. For example, the $\sim 40 \mathrm{AU}$ radius for the $\beta$ Leo disc is larger than the $23 \mathrm{AU}$ suggested by the blackbody fit. Therefore, the grains do not emit as blackbodies, but maintain a $\sim 112 \mathrm{~K}$ temperature at a greater distance from the star as expected for small grains that emit inefficiently at far-IR wavelengths. The inferred characteristic particle radius $a$ is $<\lambda / 2 \pi=16 \mu \mathrm{m}$. Future modeling work that combines Spitzer IRS spectra and submillimetre images with the Herschel data will constrain these grain properties and the spatial dust distribution (Churcher et al. 2010, in preparation).

\section{2. $\beta$ UMa}

Figure 1 shows that the disc emission around $\beta$ UMa is very compact at 100 and $160 \mu \mathrm{m}$. The disc is marginally resolved at $100 \mu \mathrm{m}$ and not resolved at $160 \mu \mathrm{m}$. The apparent asymmetry in the $160 \mu \mathrm{m}$ disc image is likely artificial; it is an effect of interpolation applied to the image at native (Nyquist sampled) resolution. The flux densities measured for $\beta$ UMa confirm the earlier 100 and $160 \mu \mathrm{m}$ detections.

The disc component of the $\beta$ UMa SED is well fit by blackbody grains with a temperature comparable to that of $\beta$ Leo, requiring a bigger disc around the more luminous star. Therefore, assuming black body grains the radial estimate is $51 \mathrm{AU}$, equivalent to the deconvolved disc radius from the 2D Gaussian fit to the $100 \mu \mathrm{m}$ image. The resolved size thus suggests an absence of small grains such as that inferred for beta Leo in Sect. 4.1. More detailed modeling will be forthcoming in a future paper.

\section{3. $\eta$ Corvi}

The new Herschel images in Fig. 1 show that $\eta$ Corvi is resolved at both 100 and $160 \mu \mathrm{m}$, as expected based on the $\sim 300 \mathrm{AU}$ submillimeter size derived by Wyatt et al. (2005). The variation in morphology from centrally peaked emission at $100 \mu \mathrm{m}$ to a double-peaked limb brightened ring at $160 \mu \mathrm{m}$ (as observed at $450 \mu \mathrm{m}$ ) is consistent with an outer cool ring filled in by warmer dust which dominates the emission at $100 \mu \mathrm{m}$. This could be evidence of the third temperature component proposed by Chen et al. (2006) and observed in $\epsilon$ Eri by Backman et al. (2009), although this was tentatively ruled out in mid-IR imaging by Smith et al. (2008), and more generally suggests the radial distribution of material is broader than the two ring system originally envisaged by Wyatt et al. (2005).

The $R_{\text {obs }}$ estimate from Table 1 is equivalent to the submillimetre size. The two intensity maxima in the $160 \mu \mathrm{m}$ image are roughly a beamwidth $\left(11^{\prime \prime}\right)$ apart, identical to the $450 \mu \mathrm{m}$ SCUBA imaging of Wyatt et al. (2005) who inferred that the emission arises from a ring at moderate inclination. Fitting a 2D Gaussian to the $100 \mu \mathrm{m}$ image of Fig. 1 gives a position angle of $102^{\circ} \pm 7^{\circ}$ and an inclination of $\sim 50^{\circ}$ from the line of sight. The position angle of the two peaks at $160 \mu \mathrm{m}$ is $\sim 135^{\circ}$. These inclinations are consistent with the $450 \mu \mathrm{m}$ measurement of $130^{\circ}$ $\pm 10^{\circ}$.

A two component model of the SED of Fig. 2 shows similar results to Wyatt et al. (2005) who found disc components of $40 \mathrm{~K}$ and $370 \mathrm{~K}$. The warm component (Smith et al. 2009, 2008; Chen et al. 2006) shown in Fig. 2 has a blackbody temperature of $346 \mathrm{~K}$, corresponding to a radial distance of $1.4 \mathrm{AU}$ from the star. The cold component has a temperature of $33 \mathrm{~K}$, corresponding to a radial separation of $160 \mathrm{AU}$ from the star, consistent with 


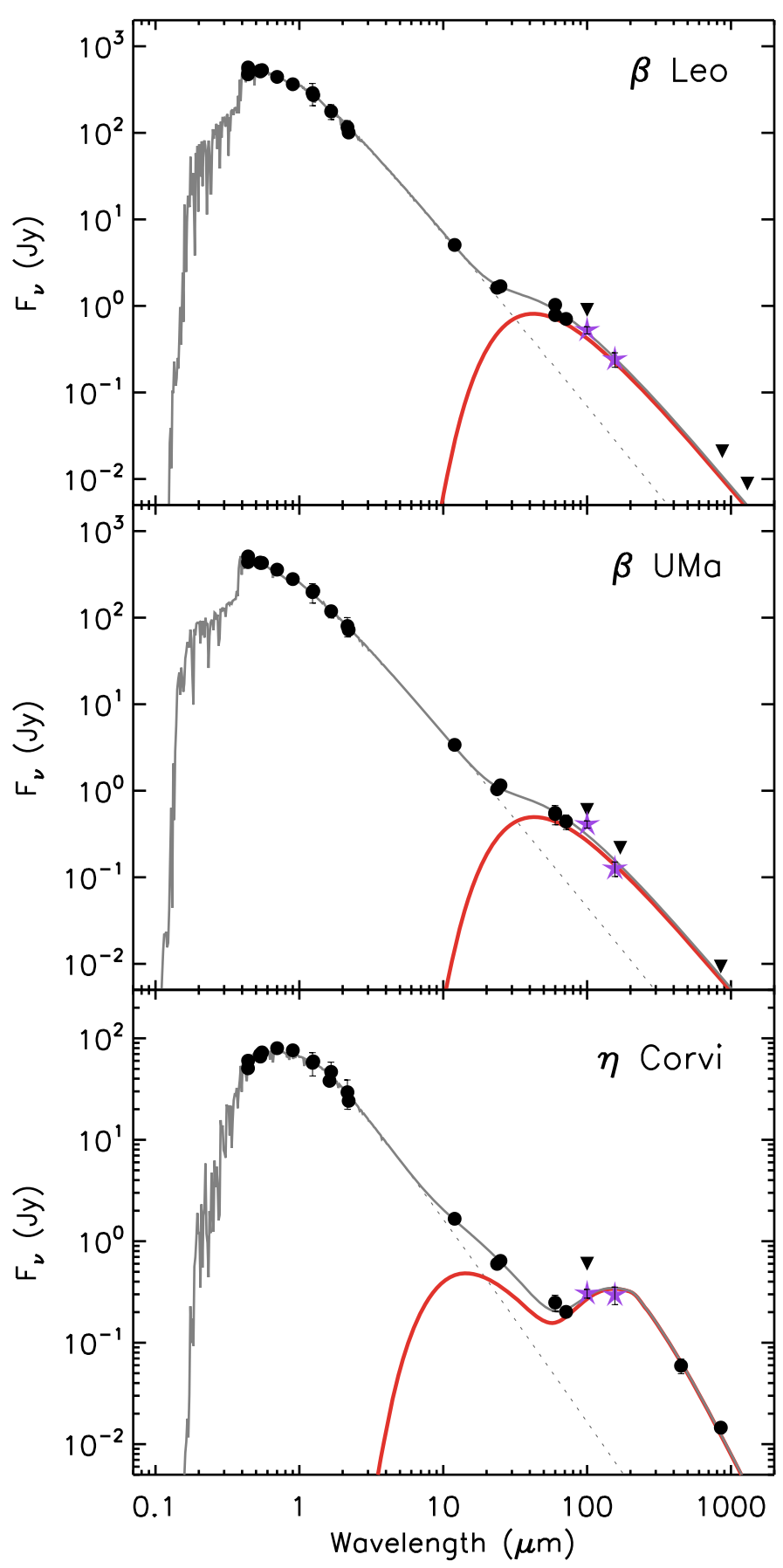

Fig. 2. Spectral energy distributions of three DEBRIS targets (grey lines): $\beta$ Leo, $\beta$ UMa and $\eta$ Corvi (top to bottom). Stars mark the 100 and $160 \mu \mathrm{m}$ flux densities from Herschel. Optical data were obtained from Simbad and Vizier. Spitzer 24 and $70 \mu \mathrm{m}$ data are from Su et al. (2006) and Beichman et al. (2006). IRAS 60 and $100 \mu \mathrm{m}$ flux densities are reprocessed through SCANPI. ISO fluxes are from Habing et al. (2001). Submillimetre photometry for $\beta$ Leo and $\eta$ Corvi are from Holmes et al. (2003) and Wyatt et al. (2005), respectively. The submillimetre upper limit for $\beta$ UMa is from the SCUBA (Submillimetre Common User Bolometer Array) archive. We use synthetic photometry to fit NextGen model atmospheres (dotted lines) assuming $\log (g)=4.5$, $[\mathrm{M} / \mathrm{H}]=0$, and no reddening. Blackbody disc models (thick red lines) are fit to star-subtracted excesses following Rhee et al. (2007). We use a modified blackbody with $210 / \lambda$ beyond $210 \mu \mathrm{m}$ for the cold component of $\eta$ Corvi, reflecting the inefficient emission from grains at (sub-)mm wavelengths (Wyatt 2008). The star and disc parameters are presented in Table 1.
$R_{\mathrm{obs}}$. As for $\beta \mathrm{UMa}$, the resolved size suggests an absence of the small grains implied for $\beta$ Leo in Sect. 4.1.

Most importantly, the images in Fig. 1 provide an estimate of the disk size at wavelengths intermediate between the submillimetre (which shows emission at $\sim 150 \mathrm{AU}$ ) and midIR (which shows emission at $<3.5 \mathrm{AU}$ ). This will be crucial for modeling the origin of the far-infrared morphology, which most resembles the submillimetre emission. Simultaneously modeling these several images will constrain in more detail the dust properties of the disc system.

\section{Summary}

These early images of known debris disc hosts highlight the resolving power of Herschel. For $\eta$ Corvi, the addition of resolved images in the FIR provides important constraints on the outer disc grain properties, and our data support the presence of a warmer inner component to the cool outer ring. We have resolved the discs around $\beta$ Leo and (marginally) $\beta$ UMa for the first time and find that both have sizes on the order of the Kuiper Belt. Both are among the smallest discs yet resolved.

The DEBRIS project will push the detection limits for debris discs around nearby stars towards Kuiper-Belt levels. Spitzer volume-limited surveys achieved an rms of about $5 \mathrm{mJy}$ at $70 \mu \mathrm{m}$ (e.g., Trilling et al. 2008), compared to our $1.2 \mathrm{mJy} \mathrm{rms}$ at $100 \mu \mathrm{m}$, where the contrast to the photosphere is generally also increased. This improved sensitivity, coupled with the improved resolution of Herschel and when applied to the large sample of discs that DEBRIS will ultimately observe, will satisfy the paucity of direct measurements of disc sizes that currently impedes modelling of debris discs.

Acknowledgements. We thank our referee, K. Stapelfeldt, for a constructive and insightful report. Support for this work, part of the NASA Herschel Science Center Key Program Data Analysis Program, was provided by NASA through a contract (No. 1353184, PI: H. M. Butner) issued by the Jet Propulsion Laboratory, California Institute of Technology under contract with NASA. This project is supported by a Space Science Enhancement Program grant from the Canadian Space Agency.

\section{References}

Backman, D., Marengo, M., Stapelfeldt, K., et al. 2009, ApJ, 690, 1522 Beichman, C. A., Bryden, G., Stapelfeldt, K. R., et al. 2006, ApJ, 652, 1674 Chamberlain, M. A., Lovell, A. J., \& Sykes, M. V. 2007, Icarus, 192, 448 Chen, C. H., Sargent, B. A., Bohac, C., et al. 2006, ApJS, 166, 351

Dominik, C., \& Decin, G. 2003, ApJ, 598, 626

Habing, H. J., Dominik, C., Jourdain de Muizon, M., et al. 2001, A\&A, 365, 545 Holmes, E. K., Butner, H. M., Fajardo-Acosta, S. B., \& Rebull, L. M. 2003, AJ, 125,3334

Ott, S. 2010, in Astronomical Data Analysis Software and Systems XIX, ed. Y. Mizumoto, K.-I. Morita, \& M. Ohishi, ASP Conf. Ser.

Phillips, N. M., et al. 2010, MNRAS, 375

Pilbratt, G. L., Riedinger, J. L., Passvogel, T., et al. 2010, A\&A, 518, L1

Poglitsch, A., Waelkens, C., Geis, N., et al. 2010, A\&A, 518, L2

Rhee, J. H., Song, I., Zuckerman, B., \& McElwain, M. 2007, ApJ, 660, 1556

Rhee, J. H., Song. I., \& Zuckerman, B., 2008, ApJ, 675, 777

Sheret, I., Dent, W. R. F., \& Wyatt, M. C. 2004, MNRAS, 348, 1282

Smith, R., Wyatt, M. C., \& Dent, W. R. F. 2008, A\&A, 485, 897

Smith, R., Wyatt, M. C., \& Haniff, C. A. 2009, A\&A, 503, 265

Su, K. Y. L., Rieke, G. H., Stansberry, J. A., et al. 2006, ApJ, 653, 675

Thébault, P., \& Augereau, J.-C. 2007, A\&A, 472, 169

Trilling, D. E., Bryden, G., Beichman, C. A., et al. 2008, ApJ, 674, 1086

Wyatt, M. C. 2008, ARA\&A, 46, 339

Wyatt, M. C., \& Dent, W. R. F. 2002, MNRAS, 334, 589

Wyatt, M. C., Greaves, J. S., Dent, W. R. F., \& Coulson, I. M. 2005, ApJ, 620, 492

Wyatt, M. C., Smith, R., Su, K. Y. L., et al. 2007, ApJ, 663, 365

Page 5 is available in the electronic edition of the journal at http://www . aanda. org 
1 Herzberg Institute of Astrophysics, National Research Council Canada, 5071 West Saanich Road., Victoria, BC, Canada, V9E 2E7, Canada

e-mail: brenda.matthews@nrc-cnrc.gc.ca

2 University of Victoria, Finnerty Road, Victoria, BC, V8W 3P6 Canada

3 UK Astronomy Technology Center, Royal Observatory, Blackford Hill, Edinburgh EH9 3HJ, UK

4 Institute of Astronomy, University of Cambridge, Madingley Road, Cambridge, CB3 OHA, UK

5 Institute for Astronomy, University of Edinburgh, Royal Observatory, Blackford Hill, Edinburgh EH9 3HJ, UK

${ }^{6}$ Laboratoire d'Astrophysique, Observatoire de Grenoble, Université J. Fourier, CNRS, France

7 Department of Astronomy, University of California, 601 Campbell Hall, Berkeley, CA, 94720, USA

8 School of Physics and Astronomy, University of St Andrews, North Haugh, St Andrews, Fife KY16 9SS, UK

9 Observatoire de Paris - CNRS, 77 Av. Denfert Rochereau, 75014 Paris, France

10 Centro de Astrobiología (CSIC-INTA), 28850 Torrejón de Ardoz, Madrid, Spain

11 Department of Astrophysical Sciences, Ivy Lane, Peyton Hall, Princeton University, Princeton NJ 08544, USA
12 Département de physique et Observatoire du Mont-Mégantic, Université de Montréal, C. P. 6128, Succ. Centre-ville, Montréal, QC H3C 3J7, Canada

13 Department of Physics and Astronomy, James Madison University, Harrisonburg, VA 22807, USA

14 ALMA JAO, Avda. Apoquindo 3846, Piso 19, Edificio Alsacia, Las Condes, Santiago, Chile

15 Thüringer Landessternwarte, Sternwarte 5, D-07778 Tautenburg, Germany

16 Astronomy Department, University of Texas at Austin, 1 University Station C1400, Austin, TX 78712-0259, USA

17 Hamburger Sternwarte, Gojenbergsweg 112, 21029 Hamburg, Germany

18 Department of Physics, Science Laboratories, Durham University, South Road, Durham, DH1 3LE, UK

19 Dept. of Physics \& Astronomy, University of California, Los Angeles, 475 Portola Plaza, Los Angeles, CA 90095-1547, USA

20 Geneva Observatory, Astronomy Department of the Geneva University, Switzerland

21 Leiden Observatory, Leiden University, Postbus 9513, 2300 RA, Leiden, The Netherlands

22 Harvard-Smithsonian Center for Astrophysics, 60 Garden Street, Cambridge, MA 02138, USA 